\title{
Prevalence, antibiotic resistance pattern and identification of extended spectrum beta- lactamase producing Klebsiella pneumoniae by phenotypic and genotypic method from various clinical samples
}

\author{
Shireen Rana', Bilal Ahmad Mir ${ }^{2, *}$, S.B. Siddesh ${ }^{3}$ \\ ${ }^{1}$ Tutor, ${ }^{2}$ Associate Professor, ${ }^{3}$ Professor and HOD, ${ }^{1}$ Dept. of Pharmacology, ${ }^{2,3}$ Dept. of Microbiology, Khaja Bandanawaz Institute of \\ Medical Sciences, Kalaburagi, Karnataka, India
}

*Corresponding Author: Bilal Ahmad Mir

Email: drbilalahmad86@gmail.com

\begin{abstract}
The present study is about ESBL producing Klebsiella pneumoniae which is diagnosed by phenotypic and genotypic methods. A total of 5055 properly collected, well labelled samples, received in the Microbiology laboratory were processed following standard protocol. Total 5055 samples, $805(16.0 \%)$ Klebsiella pneumoniae were isolated. 226/3415 (6.64\%) urine, 218/649 (33.6\%) wound, 127/342 (37.1\%) blood, 215/604 (35.6\%) pus and 18/45(40.0\%) sputum samples tested positive for Klebsiella pneumoniae. The occurrence of ESBL producers among Klebsiella pneumoniae in the current study is 293/805 (36.4\%). Highly resistant drugs for Klebsiella pneumoniae ESBL isolates were Ampicillin (100\%), Piperacillin (100\%), Amoxycillin + clavulanic acid (66.2\%). Non ESBL producing isolates were less resistant to the same antibiotics. Out of 805 isolated $285(35.4 \%)$ and $293(36.3 \%)$ were identified as ESBL producers by double disc synergy test and phenotypic confirmatory test respectively. Samples identified as ESBL producers were confirmed by E test. Detection of gene among these isolates, single bla gene could be detected in $59(20.1 \%)$, two genes in $71(24.2 \%)$ and three genes in $156(53.2 \%)$ isolates. blaCTX-M-15 was the only gene found in $8(4 \%)$ of the Klebsiella pneumoniae isolates tested, whereas blaSHV alone was found in $42(14.3 \%)$ isolates. Among the combination of two bla genes, the combination of blaSHV and blaCTX-M-15 was found in 54 (18.4\%) isolates. This study establishes that considerable genetic diversity and variations in the distribution of ESBL producers and their bla genes exist in the clinical isolates of K. pneumoniae across Karnataka.
\end{abstract}

Keywords: ESBL, Klebsiella pneumoniae, CLSI guidelines, Gene.

\section{Introduction}

Extended spectrum beta lactamases are prevalent all over the globe, and in addition, they are found in a significant percentage of Klebsiella pneumoniae strains in certain parts of the world (Florey et al, 1942; Abraham et al, 1941). Members of Enterobacteriaceae family have emerged as one of the major causes of both nosocomial and community acquired infections. Antibiotic like beta lactams (especially extended-spectrum cephalosporins and carbapenems) and flouroquinolones are the major therapeutic options to treat infections caused by the organisms of this family (Canton et al, 2008).

Klebsiella species are important opportunistic nosocomial pathogens particularly Klebsiella pneumoniae which causes infections like septicemia, urinary tract infections, wound infections, pneumonia. Due to continuous mutations of ESBLs changes in the amino acid configuration near the active site of these beta lactamases occur, which results in the development of new enzymes showing extended substrate profiles. Till now, more than 400 different ESBLs have been identified, and these are divided into three groups: TEM, SHV and CTX-M. Now the most dominant CTX-M type worldwide is CTX-M-15, which is widespread in India (Rossolini et al, 2008; Canton et al, 2012 and Canton et al, 2006).

Determination of ESBL genes, by molecular methods in bacteria that produce ESBL and their antimicrobial resistance patterns can provide applicable information about their epidemiology and risk factors related to their infections. The aim of the study is to detect extended spectrum beta lactamase producing Klebsiella pneumoniae isolated from the clinical samples by phenotypic methods and molecular method using PCR.

\section{Materials and Methods}

The present study was conducted in Khaja Banda Nawaz Institute of Medical Sciences, Gulbarga, extending over a period of 3 year from 2015 to 2018. A total of 5055 properly collected, well labelled samples, received in the Microbiology laboratory were processed following standard protocol. The various samples which were received included urine, pus, wound sputum and blood. Samples were inoculated on nutrient agar (NA), MacConkey's agar (MA), enriched media like blood agar (BA) by streak plate method under all aseptic conditions and incubated at $37^{\circ} \mathrm{C}$ for 24hours. Klebsiella pneumoniae was isolated on the basis of grams staining and were confirmed and identified to the species level by standard biochemical tests.

Antimicrobial Susceptibility Testing: The isolates were tested by the Kirby-Bauer disc diffusion method on Muller Hinton agar using 0.5 McFarland's as the turbidity standard as per CLSI guidelines.

Detection of ESBL: Klebsiella pneumoniae isolated were tested for ESBL production by CLSI recommended screening and confirmatory methods like phenotypic confirmation method and MIC using $\mathrm{E}$ test. Isolates showing inhibition zone size of $\leq 22 \mathrm{~mm}$ with Ceftazidime $(30 \mu \mathrm{g}), \leq 25 \mathrm{~mm}$ with Ceftriaxone $(30 \mu \mathrm{g})$ and $\leq 27 \mathrm{~mm}$ with Cefotaxime $(30 \mu \mathrm{g})$ were identified as potential ESBL producers and shortlisted for confirmation of ESBL 
production. Resistance to at least one of the antibiotics was considered as positive in the screening test for possible ESBL production as per 2010 CLSI guidelines. Confirmation of ESBL was done by Double Disc Synergy test and Epsilometer test (E Test).

Identification of Organism by Genotypic Criteria: ESBL producing Klebsiella pneumoniae strains detected by phenotypic methods were tested for the presence of blaTEM, blaSHV and CTX-M-15 gene by molecular methods using PCR. ESBL producing Klebsiella pneumoniae isolates were preserved in $20 \%$ glycerol trypticase soy broth and stored at $-20^{\circ} \mathrm{C}$. These isolates were sub-cultured on Mueller Hinton agar containing cefotaxime $(2 \mu \mathrm{g} / \mathrm{ml})$ and assessed for viability and purity.

Extraction of Plasmid DNA: Bacterial plasmid was extracted using PureSol Plasmid Extraction Kit (Merck GeNei, Bangalore) as per manufacturer's instructions. The isolates were grown overnight in Luria Bertani broth with cefotaxime $(2 \mu \mathrm{g} / \mathrm{ml})$ at $37^{\circ} \mathrm{C}$. In a centrifuge vial, $1.5 \mathrm{ml}$ of this culture suspension was taken and centrifuged at 9000 $\mathrm{rpm}$ for one minute and the supernatant was discarded. The pellet was re-suspended in $200 \mu \mathrm{l}$ of solution A and $10 \mu \mathrm{l}$ of lysozyme. The tubes were placed in a dry bath at $95^{\circ} \mathrm{C}$ for one minute and immediately cooled on crushed ice for five minutes. The tubes were then centrifuged at 13,000 RPM for 10 minutes and $500 \mu \mathrm{l}$ of the supernatant was transferred to another vial. To this, $500 \mu \mathrm{l}$ of isopropanol was added, mixed well and incubated at room temperature for 5 minutes. The vials were then centrifuged for 10 minutes at 13,000 RPM and the supernatant was discarded. The resulting pellet washed with $150 \mu \mathrm{l}$ cold $70 \%$ ethanol and centrifuged again at 13,000 RPM for 5 minutes. The supernatant was discarded and the pellet was air-dried. The pellet was resuspended in $25 \mu \mathrm{l}$ of solution B with $2 \mu \mathrm{l}$ of RNase A and held for five minutes. The tubes were then stored at $-20^{\circ} \mathrm{C}$ until further study.

Polymerase Chain Reaction (PCR): blaTEM and blaSHV genes were detected by PCR in two steps; first step involved screening by amplifying a part of these genes and the second step involved amplification of the complete bla gene. Apart from the blaTEM and blaSHV genes, all plasmid DNA samples were also tested for blaCTX-M-15 gene.

\section{Results}

5055 clinical samples from urine wound, blood pus sputum were tested for Klebsiella pneumoniae and 16\% samples were found to be positive for Klebsiella pneumoniae. These positive samples were further tested for ESBL production and out of 805 sample 293 (36.4\%) were ESBL producers.

Table 1 displays Isolation of Klebsiella pneumoniae from various clinical samples. From total 5055 samples 805 (16.0\%) Klebsiella pneumoniae were isolated. 226/3415 (6.64\%) urine, 218/649 (33.6\%) wound, $127 / 342(37.1 \%)$ blood, 215/604 (35.6\%) pus and 18/45(40.0\%) sputum samples tested positive for Klebsiella pneumoniae. chisquare test was done and the $\mathrm{p}$ value was found to be significant.

Table 1: Isolation of Klebsiella pneumoniae from various clinical samples $(n=5055)$

\begin{tabular}{|l|c|c|c|}
\hline \multirow{2}{*}{ Sample } & \multicolumn{2}{|c|}{ Klebsiella pneumonia } & Total samples \\
\cline { 2 - 4 } & Positive (\%) & Negative & Number \\
\hline Urine & $227(6.64)$ & 3188 & 3415 \\
\hline Wound & $218(33.6)$ & 431 & 649 \\
\hline Blood & $127(37.1)$ & 215 & 342 \\
\hline Sputum & $18(40.0)$ & 27 & 45 \\
\hline Pus & $215(35.6)$ & 389 & 604 \\
\hline$\chi 2=679.768 ; p$ value $<0.001$ & \multicolumn{4}{|l}{} \\
\hline
\end{tabular}

Table 2 shows the distribution of ESBL production of Klebsiella pneumoniae from various clinical samples. Out of 805 samples of $K$. pneumoniae isolates 293 (36.4\%) were ESBL producers. From total 227 urine, 218 wound, 127 blood and 215 pus samples tested 85 (37.4\%), 78 (35.7), 45
(35.4\%), $75(34.8 \%)$ and $75(34.8 \%)$ were positive for ESBL producing $K$. pneumoniae respectively. maximum ESBL producing isolates were obtained from urine followed by wound and blood. however in sputum out of 18 tested samples $10(55.5 \%)$ were positive for ESBL production.

Table 2: Distribution according to clinical sources of $K$. pneumoniae isolates producing ESBL

\begin{tabular}{|l|c|c|c|}
\hline \multirow{3}{*}{ Samples } & \multicolumn{3}{|c|}{ Number of ESBl producing isolates } \\
\cline { 2 - 4 } & Tested & Positive (\%) & Negative \\
\cline { 2 - 4 } & 227 & $85(37.4)$ & 142 \\
\hline Urine & 218 & $78(35.7)$ & 140 \\
\hline Wound & 127 & $45(35.4)$ & 82 \\
\hline Blood & 18 & $10(55.5)$ & 8 \\
\hline Sputum & 215 & $75(34.8)$ & 512 \\
\hline Pus & 805 & $293(36.4)$ & \\
\hline Total & \multicolumn{3}{|c|}{} \\
\hline$\chi 2=3.26 ; \mathrm{P}=0.515$ &
\end{tabular}


Table 3 displays the antibiotic resistance pattern of ESBL producing Klebsiella pneumoniae. Highly resistant drugs for $K$. pneumoniae ESBL isolates were Ampicillin $(100 \%)$, Piperacillin (100\%), Amoxycillin + clavulanic acid (66.2\%). Non ESBL producing isolates were less resistant to the same antibiotics. Significant difference in resistant pattern between ESBL and Non ESBL isolates was found in case of beta lactam drugs Amoxycillin + clavulanic acid, Gentamicin, Amikacin, Ciprofloxacin, Tobramycin, Ofloxacin, Cefuroxime, Co - trimoxazole, Ceftazidime, Ceftriaxone, Piperacillin + Tazobactam, Nitrofurantoin. The ESBL isolates of $K$. pneumoniae were highly resistant to these set of antibiotics; however, non-ESBL isolates showed relatively higher sensitivity to them. ESBL producers showed high level of resistance to cephalosporins when compared to non-ESBL producers. About $41.9 \%$ ESBL producers were resistant to Cefuroxime were as only $14.2 \%$ non-ESBL were resistant to the same drug. $34.4 \%$ and $10.1 \%$ ESBL and non-ESBL producers were resistant to Ceftazidime respectively. $31.0 \%$ ESBL and $11.1 \%$ nonESBL were found to be resistant to Ceftriaxone. The most effective drugs found in antibiotic resistance testing against $K$. pneumoniae ESBL isolates were imipenem and Aztreonam showing 0\% resistance each.

Table 3: Antibiotic resistance pattern of Klebsiella pneumonia

\begin{tabular}{|l|c|c|c|c|}
\hline \multicolumn{1}{|c|}{ Antibiotic } & \multirow{2}{*}{ Code } & $\begin{array}{c}\text { ESBL Producer }(\mathbf{n}= \\
\mathbf{2 9 3})\end{array}$ & $\begin{array}{c}\text { Non-ESBL Producer } \\
(\mathbf{n = 5 1 2})\end{array}$ & $\begin{array}{c}\text { P- value based } \\
\text { on Chi - square } \\
\text { test }\end{array}$ \\
\cline { 3 - 4 } & & $\mathbf{n}(\mathbf{\%})$ & $\mathbf{n}(\mathbf{\%})$ & $<0.001$ \\
\hline Ampicillin & $\mathrm{A}$ & $293(100)$ & $144(28.1)$ & $<0.001$ \\
\hline Pipecillin & $\mathrm{B}$ & $293(100)$ & $169(33.0)$ & $<0.001$ \\
\hline Amoxycillin + clavulanic acid & $\mathrm{C}$ & $194(66.2)$ & $79(15.4)$ & $<0.001$ \\
\hline Gentamicin & $\mathrm{D}$ & $146(49.8)$ & $126(24.6)$ & $<0.001$ \\
\hline Amikacin & $\mathrm{E}$ & $138(47.0)$ & $109(21.2)$ & $<0.001$ \\
\hline Ciprofloxacin & $\mathrm{F}$ & $138(47.0)$ & $207(40.4)$ & $<0.001$ \\
\hline Tobramycin & $\mathrm{G}$ & $132(45.0)$ & $102(19.9)$ & $<0.001$ \\
\hline Ofloxacin & $\mathrm{H}$ & $128(43.6)$ & $157(30.6)$ & $<0.001$ \\
\hline Cefuroxime & $\mathrm{I}$ & $123(41.9)$ & $73(14.2)$ & $<0.001$ \\
\hline Co-trimoxazole & $\mathrm{J}$ & $114(38.9)$ & $84(16.4)$ & $<0.001$ \\
\hline Ceftazidime & $\mathrm{K}$ & $104(35.4)$ & $52(10.1)$ & $<0.001$ \\
\hline Ceftriaxone & $\mathrm{L}$ & $91(31.0)$ & $57(11.1)$ & $<0.001$ \\
\hline Piperacillin + tazobactam & $\mathrm{M}$ & $78(26.6)$ & $16(3.1)$ & 0.561 \\
\hline Nitrofurantoin & $\mathrm{N}$ & $54(18.4)$ & $103(20.1)$ & - \\
\hline Aztreonam & $\mathrm{O}$ & 0 & 0 & - \\
\hline Imipenem & $\mathrm{P}$ & 0 & 0 & \\
\hline
\end{tabular}

Screening test and phenotypic confirmatory test for ESBL: Out of 805 isolated 285 (35.4\%) and 293 (36.3\%) were identified as ESBL producers by double disc synergy test and phenotypic confirmatory test respectively. Samples identified as ESBL producers were confirmed by $\mathrm{E}$ test (Fig.1). Detection of gene: Among these isolates, single bla gene could be detected in $59(20.1 \%)$, two genes in 71 $(24.2 \%)$ and three genes in $156(53.2 \%)$ isolates. blaCTXM-15 was the only gene found in $8(4 \%)$ of the Klebsiella pneumoniae isolates tested, whereas blaSHV alone was found in $42(14.3 \%)$ isolates. Among the combination of two bla genes, the combination of blaSHV and blaCTX-M15 was found in $54(18.4 \%)$ isolates (Table 4).

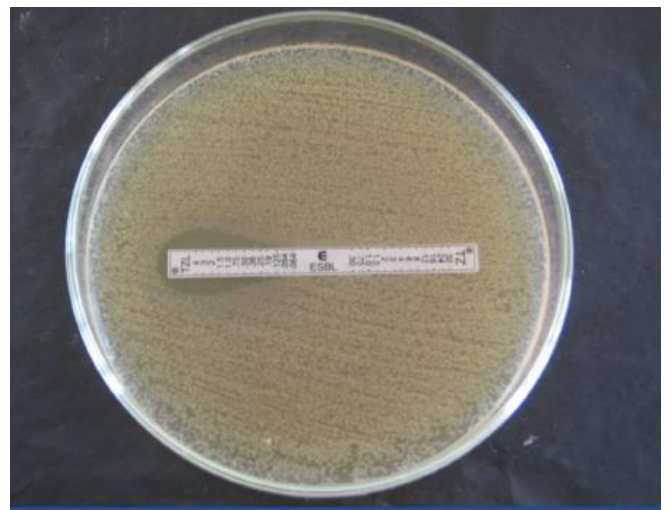

Fig. 1: E test ESBL strip showing clear cut ESBL positive organism showing Ceftazidime (TZ) MIC is reduced by $>3 \log 2$ dilutions (ratio $\mathrm{TZ} / \mathrm{TZL},>8$ ) in the presence of clavulanic acid. 
Table 4: Distribution of bla gene type in ESBL producing K. pneumonia $(\mathbf{n}=\mathbf{2 9 3})$

\begin{tabular}{|l|c|c|}
\hline \multicolumn{1}{|c|}{ bla type gene } & Positive (\%) & Negative \\
\hline TEM only & $2(0.7)$ & 291 \\
\hline SHV only & $42(14.3)$ & 251 \\
\hline CTX-M-15 only & $15(5.1)$ & 278 \\
\hline TEM + SHV & $3(1.0)$ & 290 \\
\hline TEM + CTX -M-15 & $14(5.0)$ & 279 \\
\hline SHV + CTX - M-15 & $54(18.4)$ & 239 \\
\hline $\begin{array}{l}\text { TEM + SHV+ CTX - } \\
\text { M-15 }\end{array}$ & $156(53.2)$ & 137 \\
\hline No gene & $7(2.3)$ & 286 \\
\hline$\chi 2=585.638 ; p<0.001$ & \\
\hline
\end{tabular}

\section{Discussion}

The spread of ESBL producing bacteria has become strikingly rapid worldwide, indicating that continuous monitoring systems and effective infection control measures are absolutely require. It is believed that inappropriate and rampant usage of these antibiotics has led to emergence and selection of ESBL producers (Pinto et al, 2004 and Dancer et al, 2001). Restriction in usage of these antibiotics have been shown to be associated with reduction in incidence of ESBL production (Ballow et al, 1992 and Murki et al, 2010). The occurrence of ESBL producers among Klebsiella pneumoniae in the current study was 293/805 (36.4\%) (Table 2).

Majority of the clinical samples yielding the isolates used in this study came from urine (37.4), wound (35.7\%) and blood $(35.4 \%)$ formed the bulk of the samples that yielded culture of K. pneumoniae. In 2009, CLSI introduced two-step procedure for the detection of ESBL producers. In the first step, isolates of $K$. pneumoniae should be screened for resistance to one or more of third-generation indicator cephalosporin (ceftriaxone, cefotaxime, ceftazidime).

In the present study, $35.4 \%$ of $K$. pneumoniae were found to be positive for possible production of ESBLs in the screening test. The screening test involved ceftriaxone, cefotaxime, ceftazidime and but not cefpodoxime. In the pilot study, the cefpodoxime disk did not meet the expected quality standard and therefore could not be included.

In the second step recommended by CLSI, isolates that are positive in the screening test are confirmed for ESBL production by clavulanic acid based test. Although ESBLs are inhibited by clavulanic acid, sulbactam and tazobactam, most phenotypic methods are based on clavulanic acid. In this study, both ceftazidime, ceftazidime+clavulanic acid (CAZ/CAC) and cefotaxime, cefotaxime+clavulanic acid (CTX/CEC) disks were used as per CLSI protocols. CTX/CEC disks were able to detect ESBL production in $98.6 \%$ of isolates that were positive in the screening test.

CAZ/CAC disks were able to detect ESBLs in only $89.7 \%$ of isolates, suggesting that CTX/CEC disks were more sensitive in detecting ESBLs. In this study out of 805 isolates of Klebsiella pneumoniae, 293 isolates could be phenotypically confirmed as ESBL producers by the CLSIPCT method.
Prevalence of ESBL producing $K$. pneumoniae was found to be $36.4 \%$. All ESBL producers were uniformly resistant to ceftriaxone, Ceftazidime, cefotaxime, rendering them inappropriate for treatment. None of the ESBL producers tested in this study exhibited susceptibility MIC breakpoint of $\leq 1 \mu \mathrm{g} / \mathrm{ml}$ to cefotaxime and ceftriaxone and $\leq 4 \mu \mathrm{g} / \mathrm{ml}$ to Ceftazidime. While ESBL-KP was similarly resistant to cefotaxime and ceftriaxone at MIC level of $\geq 128$ $\mu \mathrm{g} / \mathrm{ml}$ in this study, significantly more number of ESBL-KP were resistant to Ceftazidime. In studies from India, susceptibility of ESBL-KP to ceftazidime ranged from $2.5 \%$ to $70 \%$. Susceptibility of ESBL-KP to cefotaxime ranged ranged from $10.1 \%$ to $30 \%$. In few other studies, none of the ESBL producers were found to be susceptible to cefotaxime or ceftazidime (Vijayakanthi et al, 2013).

MIC90 of cefotaxime, ceftriaxone and Ceftazidime amongst all ESBL producers were $>128 \mu \mathrm{g} / \mathrm{ml}$ indicating high level resistance. In contrast to these findings, a Greek study reported slightly higher MIC values of Ceftazidime than cefotaxime (Poulou et al, 2014) among ESBL producers. Even the type of CTX-M ESBL can influence the MIC values. In a Korean study, the MIC90 of cefotaxime among CTX-M-14 and CTX-M-15 ESBL producers were $>128 \mu \mathrm{g} / \mathrm{ml}$ but differed with respect to Ceftazidime (Kim S et al, 2014). While the MIC90 of Ceftazidime was $32 \mu \mathrm{g} / \mathrm{ml}$ in CTX-M-14 producers, it was $256 \mu \mathrm{g} / \mathrm{ml}$ in CTX-M-15 ESBL producers. This difference is obviously due to the fact that CTX-M-15 can hydrolyze Ceftazidime better than CTXM- 14.

In this study, plasmids extracted from the bacteria served as templates for PCR. Of the 293 isolates, only 286 $(97.6 \%) K$. pneumoniae could be confirmed as ESBL producers by the detection of ESBL gene (Table 4). Among seven isolates none of the three ESBL genes neither there combinations were detected A positive phenotypic test could also be possible due to hyperproduction of classical beta-lactamases. Absence of ESBL gene could also be possible due to missing out on the detection of ESBL gene or the loss of encoding plasmid during storage. As reported worldwide, CTX-M-15 type ESBLs predominated $K$. pneumoniae $(81.5 \%)$ isolates in this study. SHV type ESBLs are derived from the chromosomal SHV betalactamases following one or several mutations in the active site of the enzyme. Single bla SHV gene was found in $14.3 \%$ of ESBL positive isolates of K.pneumonia were as SHV + CTX-M-15 was found in $18.4 \%$ isolates. In this study, $2(0.7 \%)$ blaTEM genes were detected among the ESBL producing $K$. pneumoniae. blaTEM genes have been detected in many studies across India but not many had characterized them by DNA sequencing, hence the actual prevalence of TEM ESBLs is unknown.

K. pneumoniae $(53.2 \%)$ isolates harboured all the three genes (blaTEM, blaSHV and blaCTX-M-15), TEM+SHV was detected in $3(1 \%)$ isolates were as TEM + CTX- M-15 was found in $14(5 \%)$ of the total isolates. Presence of multiple bla genes is advantageous to K. pneumoniae as it confers resistance to multiple class of beta-lactam antibiotics and thereby provide survival advantage. 
This study establishes that considerable genetic diversity and variations in the distribution of ESBL producers and their bla genes exist in the clinical isolates of $K$. pneumoniae across Karnataka. Such studies have to be carried on a continuous basis to detect the emergence of newer resistant strains in the region. Regional and nationallevel surveillance programs conducted on a periodic basis are vital in understanding and documenting the evolution and spread of beta-lactamase mediated resistant bacteria. Genotypic variations among the bacteria in the population may also influence the choice of strategy for their control and outcome.

\section{Acknowledgement}

We the authors are thankful to the participants, department of Microbiology, Pharmacology, IEC, Dean and principal of KBN institute of medical sciences for helping us to conduct this study.

\section{References}

1. Abraham EP, Chain E, Fletcher CM, Florey HW, Gardner AD, Heatley NG, Jennings MA (1941). Further observations on penicillin. Lancet II: 177.

2. Ballow $\mathrm{CH}$, Schentag JJ. Trends in antibiotic utilization and bacterial resistance. Report of the National Nosocomial Resistance Surveillance Group. Diagn Microbiol Infect Dis. 1992; 15:37S-42S.

3. Canton R, Coque TM. The CTX-M beta-lactamase pandemic. Curr Opin Microbiol. 2006;9:466-475.

4. Canton R, González AJM, Galán JC. CTX-M Enzymes: Origin and Diffusion. Front Microbiol. 2012;3:110-129.

5. Canton, Novais R, Valverde A, Machado E, Peixe 1, Baquero F and CoqueT M. Prevalence and spread of extended-spectrum $\beta$-lactamase producing Enterobacteriaceae in Europe. Clin Microbiol Infect. 2008;14:144-153.

6. Clinical and Laboratory Standards Institute (2010). Performance Standards for Antimicrobial Susceptibility Testing: Twenty-first Informational Supplement M100-S20. Wayne, PA, USA: CLSI.

7. Clinical and Laboratory Standards Institute (2014). Performance Standards for Antimicrobial Susceptibility Testing: Twenty-fourth Informational Supplement M100-S24. Wayne, PA, USA: CLSI.
8. Dancer SJ. The problem with cephalosporins. J Antimicrob Chemother. 2001;48:463-478.

9. Florey HW, Jennings MA. Some Biological Properties of Highly Purified Penicillin. Br J Exp Pathol. 1942;23:120-123.

10. Kim S, Sung JY, Cho HH, Kwon KC, Koo SH. Characterization of CTX-M-14- and CTX-M-15-Producing Escherichia coli and Klebsiella pneumoniae Isolates from Urine Specimens in a Tertiary-Care Hospital. J Microbiol Biotechnol. 2014;24:765-770.

11. Murki S, Jonnala S, Mohammed F, Reddy A. Restriction of cephalosporins and control of extended spectrum betalactamase producing gram negative bacteria in a neonatal intensive care unit. Indian Pediatr. 2010;47:785-758.

12. Pinto Pereira LM, Phillips M, Ramlal H, Teemul K, Prabhakar $P$ (2004). Third generation cephalosporin use in a tertiary hospital in Port of Spain, Trinidad: need for an antibiotic policy. BMC Infect Dis. 4:59.

13. Poulou A, Grivakou E, Vrioni G, Koumaki V, Pittaras T, Pournaras S. Modified CLSI extended-spectrum ß-lactamase (ESBL) confirmatory test for phenotypic detection of ESBLs among Enterobacteriaceae producing various ßlactamases. $J$ Clin Microbiol. 2014;52:1483-1489.

14. Rossolini GM, D'Andrea MM, Mugnaioli C. The spread of CTX-M-type extended-spectrum beta-lactamases. Clin Microbiol Infect. 2008;14:33-41.

15. Vijayakanthi N, Bahl D, Kaur N, Maria A, Dubey NK. Frequency and characteristics of infections caused by extended-spectrum beta-lactamase producing organisms in neonates: a prospective cohort study. Biomed Res Int. 2013;7(5):62-69.

How to cite this article: Rana S, Mir B. A, Siddesh S.B. Prevalence, antibiotic resistance pattern and identification of extended spectrum beta-lactamase producing Klebsiella pneumoniae by phenotypic and genotypic method from various clinical samples. Indian J Pharm Pharmacol. 2018;5(4):202-206. 